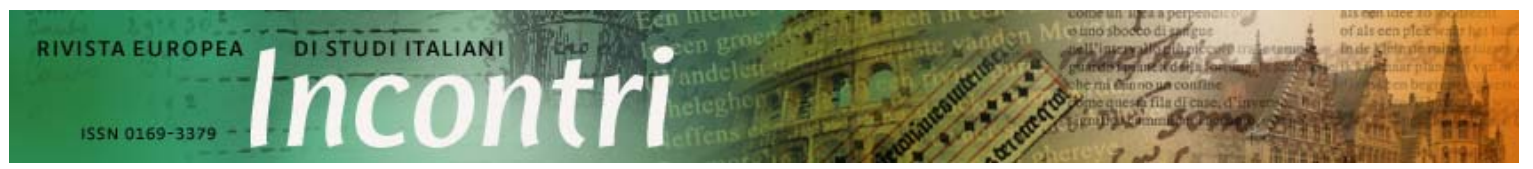

URN:NBN:NL:Ul:10-1-114249 - Publisher: Igitur publishing

Content is licensed under a Creative Commons Attribution 3.0 License

Anno 28, 2013 / Fascicolo 1 - Website: www.rivista-incontri.nl

\title{
Da campo militare a capitale: Asmara colonia italiana e oltre
}

\author{
Maristella Casciato
}

Il programma di dare all'Italia sovranità su territori esterni ai confini nazionali iniziò quasi in contemporanea con il conseguimento dell'unità politica del paese (1869), conobbe un'accelerazione all'inizio della seconda decade del Novecento e ottenne un robusto incentivo negli anni del Fascismo, per chiudersi con la caduta del regime e la sconfitta italiana nella seconda guerra mondiale. Pertanto, il quadro politico in cui la vicenda del colonialismo italiano si sviluppò, in un arco temporale di circa ottant'anni, fu assai eterogeneo.

Molto diverse furono anche le aree geografiche coinvolte nell'avventura coloniale italiana; se il Mediterraneo poteva sembrare il bacino naturale cui collegare le sorti dell'espansionismo italico, i governi scelsero di dirigersi ben più lontano, spingendosi verso le regioni del Corno d'Africa, dove non mancarono di incontrare pesanti sconfitte.

Questo saggio traccia un segmento di quella storia, concentrandosi su alcune fasi della lunga presenza degli italiani sull'altopiano eritreo e in particolare sulle vicende che accompagnarono la nascita della capitale della regione, ossia la fondazione di Asmara. Una capitale di cui si conosce poco, ma che invece rappresenta un palinsesto, seppur di provincia, di quella che fu la storia dell'urbanistica nell'Italia fra Ottocento e Novecento. Fondata su un disegno a griglia regolare, in cui si aprivano alcune piazze fiancheggiate da edifici pubblici di rilevo come il palazzo delle poste, il tribunale e le sedi delle principali banche, Asmara conobbe all'inizio del Novecento una prima espansione lungo un'asse in direzione sud-est, che raggiungeva anche gli insediamenti indigeni, costruiti in posizione separata rispetto alle aree abitate dai colonizzatori.

Con l'avvento del Fascismo, la capitale divenne una vetrina dei modi espressivi del linguaggio razionalista moderno, riconoscibili nelle architetture residenziali ma ancor più negli edifici pubblici, costruiti in numero sempre crescente: scuole, case del fascio, cinema, alberghi, palazzi per l'industria e per il commercio. L'aspetto di città moderna, pur se impregnata di motivi e modi tradizionali dell'area, rimane ancor oggi la sua cifra significativa, ed è sull'urgenza di riappropriazione di un passato recente, seppur politicamente marcato dal colonialismo, che si gioca la sfida identitaria di Asmara, capitale dell’Eritrea del XXI. secolo.

\section{Una storia lunga più di mezzo secolo}

All'Eritrea, regione del Corno d'Africa sulle rive del Mar Rosso, toccò un primato degno di nota, quello di essere la 'colonia primigenia', ufficialmente proclamata nel gennaio del 1890 , con la conseguenza che il dominio italiano su quei territori ebbe la durata più lunga 
in assoluto, che superò il mezzo secolo: un'originale specificità, se confrontata con la ben più breve vita delle successive imprese coloniali.

Passarono, infatti, più di due decenni prima che l'Italia si riaffacciasse sulla scena coloniale internazionale con l'occupazione, nel 1912, dei territori di Tripolitania e Cirenaica, bagnati dal Mediterraneo di fronte alle coste meridionali della Sicilia, e con la conquista di Rodi e di altre isole nel Dodecaneso. Dal 1925 si rafforzò la presenza italiana anche nel nord della Somalia, già in parte dichiarata colonia nel 1908, mentre nel 1934 Tripolitania e Cirenaica furono unificate per formare la 'Colonia di Libia'; l'anno successivo si procedette all'invasione dell'Etiopia.

Questa rapida escalation condusse alla proclamazione dell'impero nel maggio del 1936; contemporaneamente Eritrea, Somalia ed Etiopia furono riunite in una sola colonia, denominata 'Africa Orientale Italiana', meglio nota attraverso l'acronimo A.O.I.

Quello che sembrava un ambizioso progetto politico, diretto a restituire l'antico prestigio alla sovranità di Roma nell'ambito del bacino mediterraneo, naufragò con i rovesci militari della Seconda guerra mondiale che negli anni 1941-1943 portarono alla progressiva perdita di tutti i territori coloniali.

Alla luce di questi fatti, gli oltre cinquant'anni di presenza italiana in Eritrea meritano di essere analizzati approfonditamente, perché in quella terra si espresse il colonialismo dell'Italia liberale e perché lì si cominciarono a sperimentare modelli su cui improntare le relazioni fra colonizzatori e colonizzati, meno gravati da quell'azione propagandistica che fu il motore delle imprese avviate dal Fascismo. Infine, quel periodo ragionevolmente lungo offrì all'amministrazione italiana in Eritrea molteplici occasioni per confrontarsi con la geografia e la topografia dei luoghi, con i costumi delle popolazioni locali e con il loro habitat. Il risultato fu un processo di maturazione nell'azione di gestione della crescita urbana e territoriale che, se da un lato attinse a un modello tutto paternalistico di pianificazione, dall'altro, nell'ambito delle relazioni fra Italia e colonie, rese le realizzazioni, più che altrove, frutto di sinceri intenti di miglioramento ambientale.

Queste osservazioni non intendono certo far passare il messaggio di un'Italia benefattrice, ma solo anticipare che il governo italiano, nel campo specifico delle trasformazioni urbane, adottò nella colonia eritrea la strada del sincretismo e della contaminazione fra $i$ linguaggi, senza con questo rinunciare a progetti di modernizzazione del paese di stampo imperialistico. Tutto ciò produsse risultati di notevole efficacia sul piano economico-sociale, di cui Asmara, capitale della colonia dal 1899, fu lo specchio: città che, ancora oggi, mostra gli effetti di un felice equilibrio fra architettura, clima, culture vernacolari, modi di vita dei colonizzatori e dei colonizzati.

Eppure, nell'ambito degli studi sul colonialismo italiano, la storia della 'colonia Eritrea' non ha mai ricevuto l'attenzione riservata alle vicende che coinvolsero altre regioni conquistate dall'Italia. Questa trascuratezza da parte degli storici è durata quasi mezzo secolo, mentre un'inversione di tendenza si è prodotta dalla fine degli anni Ottanta del Novecento, con il risultato di portare a un ribaltamento della situazione storiografica. ${ }^{1}$

\footnotetext{
${ }^{1}$ Questo lavoro si pone sulla scia di rilevanti studi sull'architettura coloniale italiana avviati da un nutrito gruppo di ricercatori guidati da Giuliano Gresleri, i cui risultati sono stati resi pubblici attraverso un'esposizione accompagnata da un voluminoso catalogo. Cfr. G. Gresleri, P.G. Massaretti, S. Zagnoni (a cura di), Architettura italiana d'oltremare 1870-1940, Venezia, Marsilio, 1993. Alla conoscenza del patrimonio moderno di Asmara, realizzato dai primi anni Venti, ha concorso in modo rilevante il programma di documentazione avviato dal Cultural Assets Rehabilitation Project nel 2001, che ha prodotto l'inventario di circa 850 edifici, la catalogazione dei relativi documenti archivistici e la pubblicazione di un volume fotografico. Cfr. E. Denison (a cura di), Asmara. Africa's Secret Modernist City, London-New York, Merrell, 2003.
} 
A questo nuovo corso ha contribuito anche la rilevante fioritura del filone degli studi sul postcolonialismo, che ha prodotto testi che esaminano la vicenda coloniale eritrea partendo da una complessa lettura interdisciplinare e interculturale. ${ }^{2}$

Questa 'primavera' degli studi si è significativamente intrecciata con la lotta di liberazione del popolo eritreo che, nel 1991, ha condotto alla nascita di uno stato indipendente. In questo nuovo quadro politico si colloca anche il progetto (purtroppo rimasto sulla carta) d'iscrizione di Asmara nella World Heritage List, rivendicando la modernità dell'intervento coloniale italiano come patrimonio architettonico che appartiene di diritto alla cultura della neonata nazione eritrea.

Obiettivo di questo saggio è spiegare come lo scarto fra il passato coloniale, il presente postcoloniale e l'identità nazionale possa essere superato in nome del valore di un patrimonio architettonico riconosciuto da entrambi i soggetti e da questi condiviso.

Asmara è una città unica nella storia urbanistica del Novecento. Costruita ex-nihilo per essere capitale, presenta ancora ben riconoscibili e in ottimo stato di conservazione le testimonianze del suo passato coloniale: residenze, uffici, scuole, luoghi di culto, mercati, fabbriche, servizi pubblici e per il tempo libero, strade e altre infrastrutture. L'architettura di Asmara fu costruita attraverso forme che dovevano produrre immagini analoghe a quelle che, contemporaneamente, si andavano creando nella madre patria, il cui potere rappresentativo mostrava indubbiamente il ruolo dei colonizzatori.

Quali furono gli strumenti urbanistici e quali i modelli architettonici che permisero alla città di acquistare la sua immagine di capitale moderna? Come si sono conservate quelle testimonianze per più di mezzo secolo? Che cosa significa salvaguardare insieme il valore patrimoniale della città coloniale e la sua identità eritrea?

Per cercare di dare una risposta a queste domande è necessario fare un passo indietro e ritornare agli anni del Fascismo, durante i quali la costruzione dell'Asmara moderna ricevette il suo impulso più efficace. Più in generale è utile ricordare che il colonialismo italiano nell'Africa orientale si espresse in due successive fasi, di cui la vicenda eritrea è la più completa testimonianza: una fase, compresa fra l'ultimo decennio del XIX. secolo e il 1922, che corrisponde ai governi liberali e ai primi albori del Fascismo; una seconda attraversata dall'egemonia mussoliniana, la cui apoteosi si raggiunse nel 1936 con la proclamazione dell'impero.

L'enfasi con cui Mussolini rivendicava l'urgenza morale di un'azione espansionistica nelle terre dell'Africa nord-orientale offre un chiaro segnale di quel delicato passaggio politico che vide un giovane stato nazionale trasformarsi in una nazione imperiale. 'E $\mathrm{E}$ un popolo di 44 milioni di anime, contro il quale si tenta di consumare la più nera delle ingiustizie: quella di toglierci un po' di posto al sole'. ${ }^{3}$

Sul piano dell'azione urbanistica e di costruzione dell'architettura coloniale, Mussolini ebbe chiaro il disegno di indirizzare l'arte di governo del territorio verso un concetto totalitario e imperiale, espressione della civiltà conquistatrice. È chiaro che all'epoca era in gioco 'da un lato il rapporto fra l'architettura e la politica, dall'altro il ruolo dell'architetto'; quest'ultimo - 'interprete attraverso forme e immagini' - diventava

\footnotetext{
${ }^{2}$ Si veda R. Ben-Ghiat, M. Fuller (a cura di), Italian Colonialism, New York, Palgrave Macmillan, 2005. Cfr. numero monografico di Aut Aut, intitolato II postcoloniale in Italia, 349, gennaio-marzo 2011.

3 Enfasi dell'autore. Lo slogan si riverbera nella pubblicistica e nella letteratura sia dell'epoca, sia successiva. A titolo d'esempio cito due testi, apparsi a più di mezzo secolo di distanza, entrambi debitori del successo mediatico di quell'affascinante sineddoche: quello di Luigi Federzoni, già Ministro delle Colonie all'epoca della Marcia su Roma, A. O.I. II 'posto al sole', Bologna, Zanichelli, 1936; P. Palumbo (a cura di), A Place in the Sun. Africa in Italian Colonial Culture from the Unification to the Present, Berkeley, University of California Press, 2003.
} 
uno dei protagonisti di un disegno di governo i cui contenuti politici si intrecciavano appieno con quelli dello sviluppo della città coloniale. ${ }^{4}$

Queste ultime considerazioni sono solo parzialmente riconducibili all'esperienza eritrea, dove la politica dell'indirect rule adottata dai governi liberal-nazionali generò uno scarto fra la colonia reale, lontana dal centro, e la sua percezione in seno ai governi centrali. Paradossalmente, si svilupparono in Eritrea esperienze di disegno del territorio e dell'architettura delle città che, data la strategia di diversificazione culturale rispetto all'ambiente antropico locale, perseguita dai colonizzatori, e la totale assenza, in quel territorio, delle memorie della romanità cui l'architettura fascista altrove si ispirò, furono etichettate come 'provinciali'. Pur nel loro essere architetture 'di periferia', si trattò di prove affatto marginali, e per nulla 'fallimentari', come troppo frettolosamente si è scritto, almeno a sentire le voci di chi vi abitava.

Continuano a sorprendere le testimonianze degli italiani residenti ad Asmara negli anni Trenta, che esprimono nelle loro parole uno stato d'animo assai condiviso fra colonizzati e colonizzatori: 'una bella città, popolata da razza mista, italiani e africani. C'era molto traffico, negozi e tutto ciò che desideravamo', e ancora 'si stava veramente bene. Era una grossa cittadina, con molti cinema, ristoranti ${ }^{5}$ (Fig.7).

Questo saggio intende confrontarsi con questo intreccio di storie, con l'obiettivo di mettere in luce i momenti salienti dello sviluppo architettonico di Asmara, momenti che ne fissarono l'identità modernista, per poi ritornare, nelle conclusioni, al suo valore attuale di patrimonio.

\section{Asmara: da villaggio a città}

Fu Francesco Crispi, allora capo del governo liberale, ad assegnare a quell'avamposto italiano nel Corno d'Africa il nome di Eritrea, facendo appello al greco erythros, che significa rosso, un chiaro riferimento al colore della sabbia lungo le spiagge dell'omonimo mare.

L'amministrazione della colonia fu affidata ai militari; Massaua, città portuale sul Mar Rosso, fu dichiarata capitale nel 1891. Occupata dal generale Baldissera nell'agosto del 1889, l'area in seguito denominata Asmara, era indicata nei documenti ufficiali come 'villaggio'; per gli indigeni si trattava del senfer, ossia il 'campo'.

La costruzione di un forte militare diede origine alla nascita del primitivo insediamento, denominato 'campo cintato'. Si trattava di povere casette con giardinetti per la coltivazione di ortaggi, affiancate da pochissime costruzioni che accoglievano le funzioni amministrative, una scuola e un'infermeria. All'esterno del perimetro del 'campo cintato', ben ordinate e allineate in file parallele, vi erano le capanne tradizionali con il tetto in paglia (gli agdo), dove erano alloggiati i soldati eritrei che prestavano servizio nell'esercito italiano, gli askari.

La posizione del 'campo cintato' rispetto al principale percorso che attraversava l'altopiano di Asmara, ossia la strada carovaniera proveniente da Massaua, determinò la creazione di una polarità a scala territoriale, la cui importanza fu confermata in tutti i successivi piani di sviluppo. Arrivando dalla costa, il percorso proveniente da est tagliava rettilineo la pianura verso ovest, attraversava l'accampamento militare indigeno, dividendolo in due porzioni uguali, prima di raggiungere la collinetta del 'campo cintato'.

\footnotetext{
${ }^{4}$ G. Ciucci, 'Architettura e urbanistica. Immagine mediterranea e funzione imperiale', in: Gresleri, Massaretti, Zagnoni, (a cura di), Architettura italiana d'oltremare 1870-1940, cit., p. 109.

${ }^{5}$ Memorie orali raccolte in I. Taddia, La memoria dell'Impero. Autobiografie d'Africa orientale, Manduria, Lacaita, 1988.
} 
Sul finire del 1897 si verificò un indicativo cambiamento di prospettiva nel governo della colonia. Ferdinando Martini, letterato, scrittore e deputato al Parlamento, fu nominato primo governatore civile dell'Eritrea. Due anni dopo la sua nomina egli trasferì la capitale da Massaua ad Asmara, dando a quello spostamento il valore simbolico di un atto di fondazione. Martini resta, di fatto, colui che gettò le basi del disegno della città novecentesca. ${ }^{6}$

La crescita di Asmara fu regolata da uno schema di piano regolatore, approvato nel 1902, che comprendeva l'area a est del 'campo cintato' fino al torrente Mai Belà (proveniente da nord-ovest e piegato in direzione sud-est), che per molti anni avrebbe segnato la linea di confine della prima espansione residenziale destinata ai cittadini europei.

Il primo nucleo dell'espansione residenziale e terziaria si sviluppò su un disegno a griglia; era delimitato da due arterie principali, fra loro parallele: in alto il Corso del $\mathrm{Re}$ e, tre isolati più in basso, il Viale della Regina.

Sul Corso del Re, che seguiva la direzione dell'antica strada carovaniera, si apriva un'ampia piazza, denominata Piazza del tribunale (poi Piazza Roma), che fungeva da cuore della vita cittadina. Caratterizzata da un bel giardino con palme e fontana al centro, la piazza era circondata da edifici simbolo del potere politico ed economico: il palazzo del tribunale e le sedi delle banche centrali. Alle spalle della massiccia mole del tribunale si apriva la piazzetta delle poste, così chiamata per la presenza dell'omonimo edificio. Il sistema delle due piazze e della scacchiera suggeriva quella 'lezione di civiltà', derivante dalla geometria del piano, che fini per contraddistinguere una posizione di sudditanza della popolazione indigena, che da quel momento fu ridotta a 'suddito coloniale'.

Dal 1908 la crescita di Asmara fu regolata, per la prima volta, da una disposizione urbanistica che divideva la città in quattro zone, in conformità a una distinzione etnica che si coniugava a programmi funzionali. Erano previste la zona europea, quella mista, quella indigena, e una suburbana a vocazione industriale.

$\mathrm{Fu}$ in questa situazione, mentre si consolidava l'occupazione della Libia e contemporaneamente s'istituiva il Ministero delle Colonie, che arrivò in Eritrea l'ingegnere, di origine ligure, Odoardo Cavagnari. ${ }^{8}$ Siamo nel 1912 e il secondo governatore Salvago Raggi aveva già portato a termine una bella impresa: l'ardita linea ferroviaria proveniente da Massaua aveva raggiunto Asmara.

Cavagnari era stato nominato alla direzione dell'ufficio tecnico municipale e in quella veste mise mano alla redazione del piano regolatore generale (Fig. 8). Il disegno di quel piano, approvato nel 1914, poggiava sui nuovi assi est-ovest che correvano ai bordi

\footnotetext{
${ }^{6}$ N. Labanca,'Ferdinando Martini in Eritrea, 1897-1907. Per il riesame di un mito del colonialismo italiano', numero monografico su Ferdinando Martini, in: Farestoria, 17 (1991), pp. 26-42. Dello stesso autore, Oltremare. Storia dell'espansione coloniale italiana, Bologna, Il Mulino, 2002. Cfr. anche G. Gresleri,'Asmara in Eritrea: dal Palazzo alla Città', in: G. Gresleri, P.G. Massaretti, Architettura italiana d'oltremare, atlante iconografico, Bologna, Bononia University Press, 2009, pp. 11-16, e A. Bortolotti, 'Cesare Spighi e il Palazzo perduto', Ibidem, pp.17-19.

7 Sui temi dell'organizzazione civile del territorio in rapporto alla politica razziale cfr.: il saggio di S. Zagnoni, 'L'Eritrea delle piccole città 1897-1936', in: Gresleri, Massaretti, Zagnoni, (a cura di), Architettura italiana d'oltremare 1870-1940, cit., pp. 145-163; il bel saggio di G. Barrera,'The Construction of Racial Hierarchies in Colonia Eritrea. The Liberal and Early Fascist Period (1897-1934)', in: Palumbo (a cura di), A Place in the Sun, cit., pp. 81-115.

${ }^{8}$ Odoardo Cavagnari (Busalla di Genova 1868 - Asmara 1920) era un funzionario del Genio civile, chiamato a Roma dopo l'insediamento della capitale per partecipare alla costruzione dei nuovi edifici governativi, in particolare i Ministeri. Nel 1912 si trasferì ad Asmara; negli anni eritrei fu responsabile della redazione di altri quattro piani regolatori (Cheren, Massaua, Nefasit e Adi Ugri), oltre a quello della capitale.
} 
della precedente espansione: il Corso Italia (oggi Harnet Avenue) in basso, e il Viale Manzoni (oggi Afabet Avenue) in alto. Da questi si dipartiva un sistema di strade diagonali su cui, tenendo conto dell'accidentata orografia della piana, si pensava di impostare le nuove lottizzazioni. La rigidità della griglia era negata dal disegno di tre grandiosi patte d'oie disposti ai vertici del triangolo, che conteneva il primo nucleo della Asmara novecentesca.

Ottemperando ai criteri di separazione razziale dettati dal governo centrale, alle popolazioni autoctone non era consentito di abitare nella zona europea. Il nuovo piano non prevedeva di trattare il disegno dell'insediamento indigeno, ma si limitava a indicare una zona in posizione nord-orientale, la cui separatezza sarebbe derivata sia dall'uniformità delle forme architettoniche, sia dalla forte densità del costruito.

\section{Lungo il Viale Mussolini}

L'avvento del Fascismo non ebbe immediate ripercussioni in Eritrea. ${ }^{9}$ Forse il cambiamento più evidente si riscontrò nella denominazione del Corso Italia, che divenne Viale Mussolini ${ }^{10}$ (Figg. 2, 5).

Si tratta di quell'ampio boulevard, reso più esotico dalla presenza di un doppio filare di palme, che era stato pensato per diventare la spina dorsale della città pubblica novecentesca. Su questo percorso, lungo poco più di un chilometro, si allinearono, nei due decenni del regime fascista, alcuni degli edifici cui si deve il volto razionalista di Asmara. Anche in colonia, quindi, l'architettura moderna del razionalismo internazionale si guadagnò il posto di 'arte di Stato' (Figg. 3, 6).

Il Palazzo Falletta, un elegante blocco per appartamenti (con esercizi commerciali al piano terra), fu realizzato nel 1937-38 su disegno di Carlo Marchi e Giuseppe Cane. Il primo fu un progettista molto attivo nella città eritrea negli anni Trenta, noto soprattutto per alcuni edifici residenziali, caratterizzati dalla ricerca di una volumetria che enfatizzava la soluzione dell'angolo: ad esempio la curva concava che raccoglie e distribuisce il ritmo delle arcate nel Palazzo Gerasadik (sul Corso del Re, oggi Nafka Avenue) o l'accento espressionista della convessità del prospetto sull'attuale Denden Street. ${ }^{11}$

Negli appartamenti Falletta, Marchi propose una ricercata soluzione del palazzo urbano che richiamava i caratteri delle architetture novecentiste di Muzio: si noti la griglia

\footnotetext{
${ }^{9}$ Nel fascicolo Italian Colonies che l'Ente Nazionale Industrie Turistiche pubblicò alla fine degli anni Venti per pubblicizzare i territori d'oltremare come meta per le vacanze, la colonia Eritrea ha una presenza pari a quella di altre regioni che, sul piano paesaggistico e naturalistico, potevano vantare condizioni certamente più favorevoli.

${ }^{10}$ Mia Fuller nel suo Moderns Abroad: Architecture, cities and Italian imperialism, London-New York, Routledge 2007, traccia un quadro assai preciso delle componenti teoriche e tecniche che permisero lo sviluppo di un'architettura e di una pratica urbanistica moderne nelle colonie italiane in terra d'Africa.

${ }^{11}$ Fra le architetture progettate da Marchi va citata la sede della casa automobilistica Lancia, fatto realizzare dal più importante imprenditore dell'epoca, Salvatore Falletta. L'alta torre vetrata, che segna l'ingresso principale dell'edificio e contrasta con la forte orizzontalità degli adiacenti volumi, presenta motivi quasi futuristi, che evocano le architetture di Angiolo Mazzoni. Di fronte all'edificio Lancia, che oggi ospita una fabbrica, il coevo palazzo dell'ufficio d'igiene (trasformato in un garage) dimostra come alcuni motivi formali del razionalismo italiano, anche in questo caso la torre con orologio che sovrasta la curvilinea pensilina dell'ingresso, fossero entrati nel linguaggio corrente degli architetti colti. Si veda a questo proposito anche l'edificio della Fiat Tagliero, che si apparenta strettamente con la vocazione modernista espressa nel Manifesto dell'architettura aerea, che Mazzoni aveva pubblicato nel 1934 con Filippo Tommaso Marinetti e Mino Somenzi. Sulle architetture asmarine, cfr il saggio dell'autore 'De koloniale stad', in: $M$. Dings (a cura di), de STAD, Rotterdam, Uitgeverij 010, 2006, pp. 153-172; e inoltre, Sandro Raffone, Eritrea Razionalista, Napoli, Giannini Editore, 2010 e Sandro Raffone,'Imparando dall'Eritrea', in: I quaderni de II Cerchio, 4 (2010), pp. 23-114.
} 
che disegna le torri angolari e l'enfasi nel disegno delle aperture, tutte uguali e incorniciate come fossero quadri in una esposizione. Nella corte, i corpi scala posti nei quattro angoli diventano torricini vetrati, riecheggiando le soluzioni già sperimentate a Roma da De Renzi nel grande complesso di viale XXI Aprile e da Sabbatini negli alberghi suburbani alla Garbatella.

Il Palazzo Falletta confina con i complessi della cattedrale e della missione cattolica, che risalgono al 1923. Seguono, sullo stesso lato della cattedrale - fra l'incrocio di quello che in origine era il torrente Mai Belà, interrato alla fine degli anni Trenta $^{12}$ e l'ampia arteria perpendicolare su cui si apre il mercato delle verdure e del pesce e che termina con la grande moschea - due edifici che marcano significativamente la funzione terziaria di questo settore del viale: il palazzo del Lloyd, oggi Ministero del Turismo, e quello in cui è inserito il cinema Impero.

Le numerose sale cinematografiche costituiscono una cifra originale della modernità asmarina, sia per gli aspetti tecnico-funzionali, sia per l'accuratezza del dettaglio nel disegno degli interni e degli arredi. Mentre le denominazioni tentano di trasferire $\mathrm{i}$ caratteri di una moderna 'romanità' nell'ambito di una cultura indigena sempre più aperta alle contaminazioni.

La costruzione del cinema Impero, inserito all'interno di un isolato di vaste dimensioni, risale al 1937-38. Il disegno del prospetto principale, scandito da tre decorativi riquadri rettangolari al cui interno sono inserite aperture a oblò, propone, come si nota anche in altri edifici per residenze e negozi, la citazione di un'icona della romanità, quel sepolcro del fornaio Eurisace presso Porta Maggiore, alla cui riscoperta il Fascismo aveva dato grande enfasi. L'intonaco di colore porpora intenso e la monumentale scritta a rilievo, in caratteri lapidari, enfatizzano il suo ruolo di landmark urbano.

\section{La città postcoloniale e la sfida patrimoniale}

Quelli citati sono solo alcuni esempi di un patrimonio moderno assai unico e originale. Come si è modificata, nella coscienza collettiva, la percezione del loro valore patrimoniale? (Fig. 1)

Già con la proclamazione dell'Impero gli equilibri sociali che governavano Asmara mutarono radicalmente. La città, retroterra per le operazioni militari in Etiopia, vide crescere vertiginosamente la sua popolazione. Dai 18.000 abitanti negli anni Venti, di cui meno di un terzo erano italiani, si passò ai quasi centomila nel 1936, il 50\% dei quali giunti dall'Italia. Contemporaneamente, si assistette al costante incremento della popolazione indigena, richiamata dalla richiesta di manodopera, che andò a occupare parti sempre maggiori del vecchio centro città, mentre da parte degli italiani si era manifestata una marcata preferenza per i più salubri e meno popolosi quartieri a villini; ne è un esempio lo sviluppo accelerato del quartiere residenziale di Ghezza Banda, in direzione sud-est (Fig. 4).

Le relazioni fra colonizzatori e popolazioni autoctone divennero più critiche al momento dell'emanazione, nel giugno del 1938, delle leggi razziali. Di conseguenza nel nuovo piano regolatore, affidato nello stesso anno a Vittorio Cafiero, fu inserito un vasto

\footnotetext{
${ }^{12}$ Alla realizzazione di quest'opera di ingegneria idraulica fu data grande importanza non solo per gli aspetti tecnici del progetto, ma anche perché le fu assegnato un valore simbolico, che si fondava sul mito della dinastia salomonica dei sovrani eritrei. L'interramento del corso d'acqua fu voluto dal governatore Giuseppe Daodiace, uomo forte del fascismo, giunto in Eritrea alla fine del 1937 dalla Libia. Cfr. F. Monile, 'Una vecchia leggenda e una nuova volta di cemento armato', in: Italia d'Oltremare, 12 (1938), p. 236.
} 
quartiere indigeno, in direzione nord-est, posto di là della collina di Abbashaul, la quale aveva la funzione di barriera verde.

Allo scoccare della Seconda guerra mondiale gli eventi militari precipitarono presto; la prima incursione aerea degli inglesi su Asmara data al giugno del 1940; nell'aprile del 1941 la capitale fu occupata e passò nelle mani dell'amministrazione militare britannica.

Gli italiani furono autorizzati a continuare a svolgere le attività di amministrazione civile e si visse un periodo di vivacità nelle attività produttive, come testimoniato dalla mostra che si inaugurò nel 1943 e dalla costituzione, l'anno successivo, della Camera di Commercio. Alla fine degli anni Quaranta, sui quasi 130.000 abitanti di Asmara, vi erano ancora più di 17.000 italiani rimasti.

Le contraddizioni maturate in quel periodo furono alla base della singolare struttura sociale che la città visse per un lungo periodo. La cultura dello zoning, tipica del disegno classista delle città occidentali, era già stata riadattata nei primi anni Trenta da una società articolata e moderna, che aveva fatto di Asmara una capitale imprenditoriale. Anche a prescindere da quella zona mista in cui indigeni ed europei si trovarono a convivere porta a porta, i piani per Asmara non avevano dato origine a un organismo urbano basato su una decisa discriminazione; così, in mancanza di memorie separate, la città coloniale è diventata in larga misura la città degli abitanti.

Cosi rispondeva Maria, un'italo-eritrea asmarina, a Giulia Barrera che la intervistava: 'Solo durante il fascismo ci fu un feroce razzismo. Prima del 1935 gli Italiani mantennero un atteggiamento umano e amichevole. Certamente non portavano le nostre madri al ristorante o al cinema. La separazione c'era, ma era come naturale, non era imposta'. ${ }^{13}$

La questione della memoria del colonialismo si presenta, come fa notare Nicola Labanca, assai complessa, tenendo conto dell'iniziale distinzione fra quanti ebbero un'esperienza diretta (e forse non sono mai guariti dal mal d'africa) e quanti vissero il colonialismo solo attraverso la propaganda. ${ }^{14}$ In particolare nel caso della 'colonia primogenita', la vicenda presenta numerose anomalie, se così consideriamo, ad esempio, il fatto che la pratica di governo continuò a farsi carico delle diverse tradizioni locali, delle stratificazioni sociali all'interno dei villaggi e del continuo pendolo fra amministrazione centrale ed etno-geografie. In questo senso, la tendenza a non voler abbandonare il luogo comune di uno slogan come 'italiani brava gente' è ancora assai presente nella memoria collettiva del colonialismo. Un vero cambiamento e invece presente nella storiografia che si è sviluppata dagli anni Novanta, le cui potenzialità sono forse ancora da mettere completamente a fuoco.

Assai diversa, affatto nostalgica e non per questo meno ambigua, la posizione della gente di Asmara e quella di coloro che vissero la diaspora eritrea in Italia. Si tratta di un capitolo complesso e che merita uno studio ad hoc; ${ }^{15}$ a me sembra importante ricordare che la cosiddetta 'Casa degli Italiani', nel cuore di Asmara, continua tutt'oggi a essere un luogo di incontro multietnico, per tutte le generazioni di asmarini e non solo. Non meno indicativo di questo trascorso in colonia, la presenza di molte parole italiane che ancora resistono incastrate nella lingua tigrina o la riapertura di alcune scuole italiane, dove gli

\footnotetext{
${ }^{13}$ Barrera, 'The Construction of Racial Hierarchies in Colonia Eritrea', cit., p. 81.

${ }^{14} \mathrm{~N}$. Labanca, 'History and Memory of Italian Colonialism Today', in: J. Andall\& D. Duncan (a cura di), Italian Colonialism: Legacy and Memory, Bern, Peter Lang, 2005, p. 29-46.

${ }^{15}$ Se ne trova traccia nelle testimonianze sui diversi aspetti della vita quotidiana nella capitale nel film Asmara Eritrea, diretto da Caterina Borelli, Anonymous Production, 2007, distribuito da DER-Documentary Educational Resources, USA.
} 
studenti traducono Dante nella loro lingua madre, come documentato in un affascinante viaggio radiofonico intitolato Le Parole dell'Eritrea. ${ }^{16}$

\section{Conclusioni}

Il riferimento a un colonialismo paternalistico non serve, ovviamente, a mitigare il disegno politico imperialista che l'urbanistica e l'architettura hanno interpretato in Eritrea. La sua comprensione aiuta, piuttosto, a indirizzare l'Eritrea postcoloniale verso un modello di gestione articolata del processo di valorizzazione del suo patrimonio architettonico.

Vale la pena ricordare come la storiografia recente abbia sempre più spesso messo l'accento sulla specificità del caso eritreo tra il colonialismo italiano. Sul piano storicopolitico l'osservazione di Giampaolo Calchi Novati, 'Eritrea as such did not exist before Italian Eritrea, ${ }^{17}$ spiega come la contemporaneità della storia eritrea se confrontata, ad esempio, con il peso del patrimonio storico etiope, abbia permesso un'assimilazione quasi diretta del lascito architettonico dei colonizzatori. Questo si è dimostrato essere un carattere vincente anche in occasione della più recente lotta per la liberazione, tenendo conto del fatto che la nuova identità eritrea ha potuto affrancarsi dall'esperienza coloniale continuando a considerarla integrata alla sua stessa storia come nazione.

Oggi Asmara è una capitale africana del XXI. secolo che conserva pressoché intatti i caratteri urbani del programma di colonizzazione avviato dall'Italia a fine Ottocento. Abbandonata la koiné eclettica e regionalista, l'architettura asmarina fu pienamente investita dalla rivoluzione razionalista e dai linguaggi modernisti che si stavano affermando in campo internazionale, ben al di là dell'Italia fascista. ${ }^{18}$ Ovviamente i progettisti declinarono la forma e l'immagine di quelle architetture sull'onda di una Italianness di provincia, depurandole da ogni guizzo di avanguardismo, e indirizzando, piuttosto, il progetto verso un elementarismo che meglio si addiceva a un knowhow tecnico-costruttivo di stampo prettamente locale e artigianale.

Il caso di Asmara s'iscrive, dunque, in un processo di decolonizzazione ormai irreversibile, ma che deve ancora trovare le strade per adottare una politica di riconoscimento di un'eredità condivisa, svincolata dalle motivazioni che la generarono e insieme capace di coglierne le potenzialità e di sfruttarle per la definizione di quella riconquistata identità locale nell'ambito della sfida globale.

\footnotetext{
${ }^{16}$ Le Parole dell'Eritrea, radio documentario di Alessandro Bosetti su Radio 3, Tre Soldi, 22-25 ottobre 2012.

${ }^{17} \mathrm{G}$. Calchi Novati, 'National Identities as a By-Product of Italian Colonialism: A Comparison of Eritrea and Somalia', in: Italian Colonialism: Legacy and Memory, cit., p. 56.

${ }^{18}$ M. Casciato, 'Une place au soleil: le patrimoine colonial italien d'Asmara en Érythrée', in: Architecture coloniale et patrimoine. Expériences européennes, Paris, Institut National du Patrimoine, 2006, pp. 103111.
} 


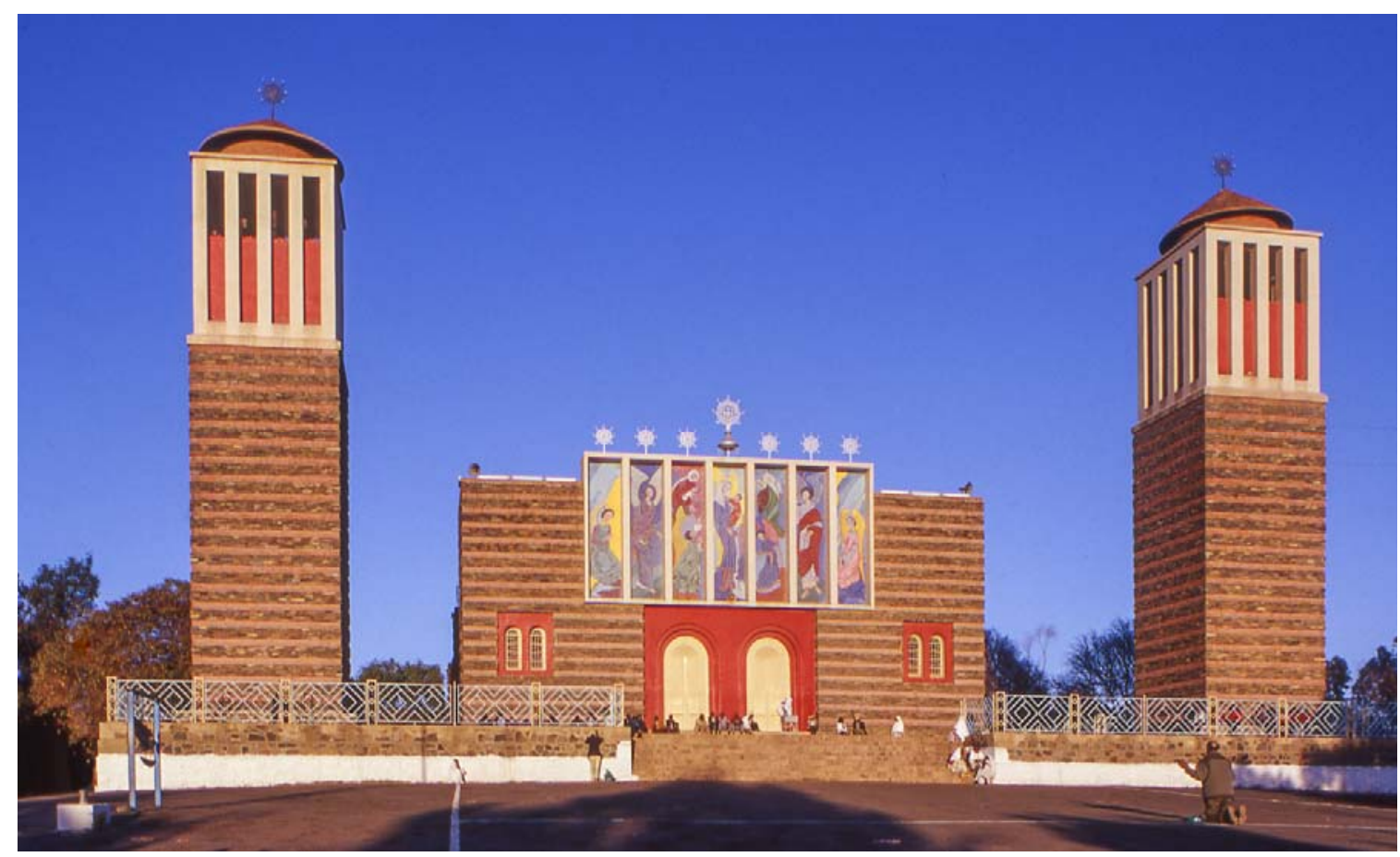

Fig. 1 @ 2013, Maristella Casciato. La chiesa copta di Bièt Christiàn (nota come la cattedrale di Enda Mariam), costruita fra fine Trenta e inizio Quaranta in sostituzione di quella realizzata intorno al 1920 dall'architetto Ernesto Gallo.

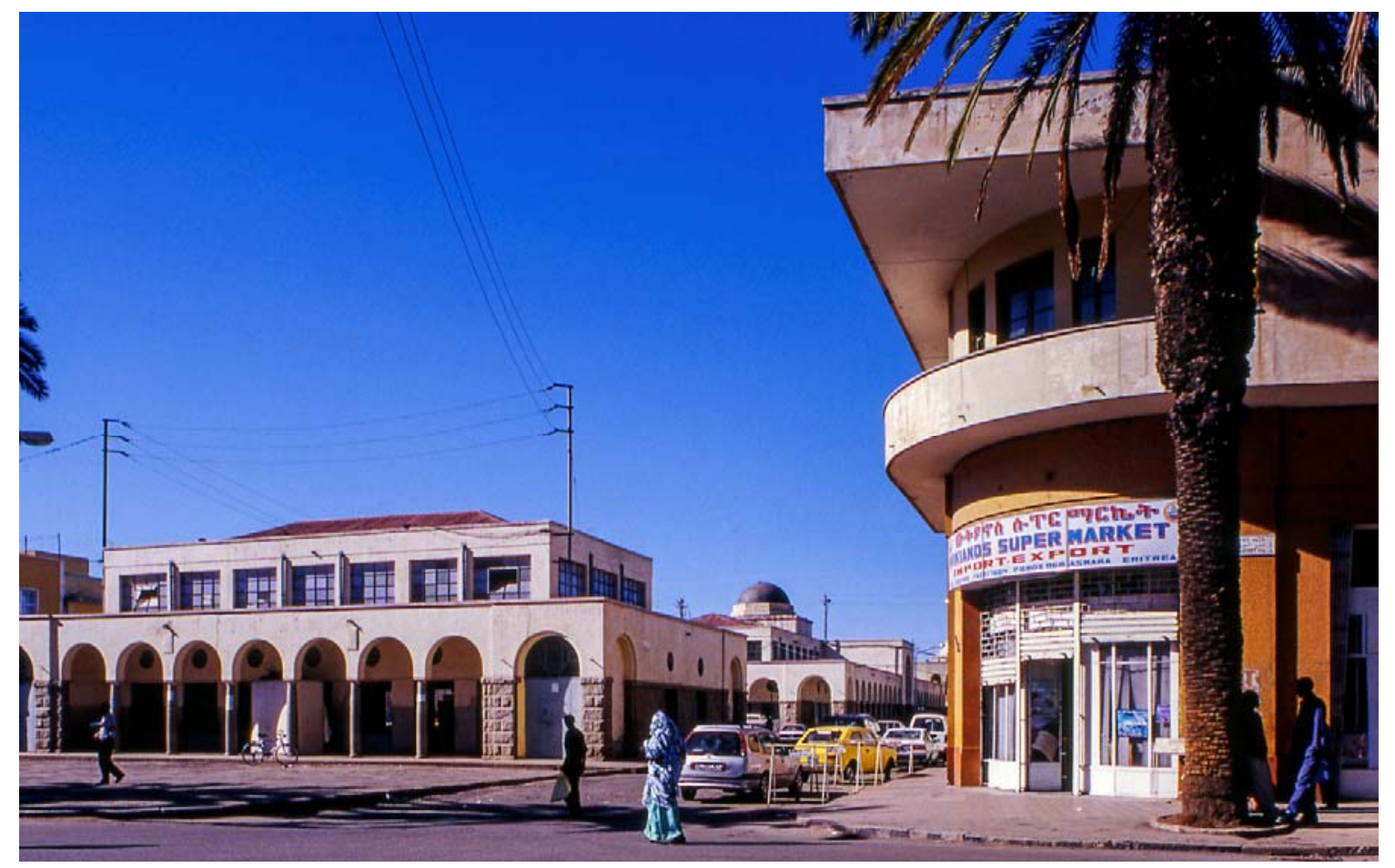

Fig. 2 ( 2013, Maristella Casciato. Edificio per appartamenti e negozi (arch. Antonio Vitaliti, 1944 ca.) su Harnet Avenue all'angolo con la piazza del mercato. La lunga piazza, perimetrata da edifci su due piani porticati, fu realizzata su disegno di Guido Ferrazza, con Ferruccio Mazzanti e Giuseppe Arata, fra il 1938 e i primi anni Quaranta 


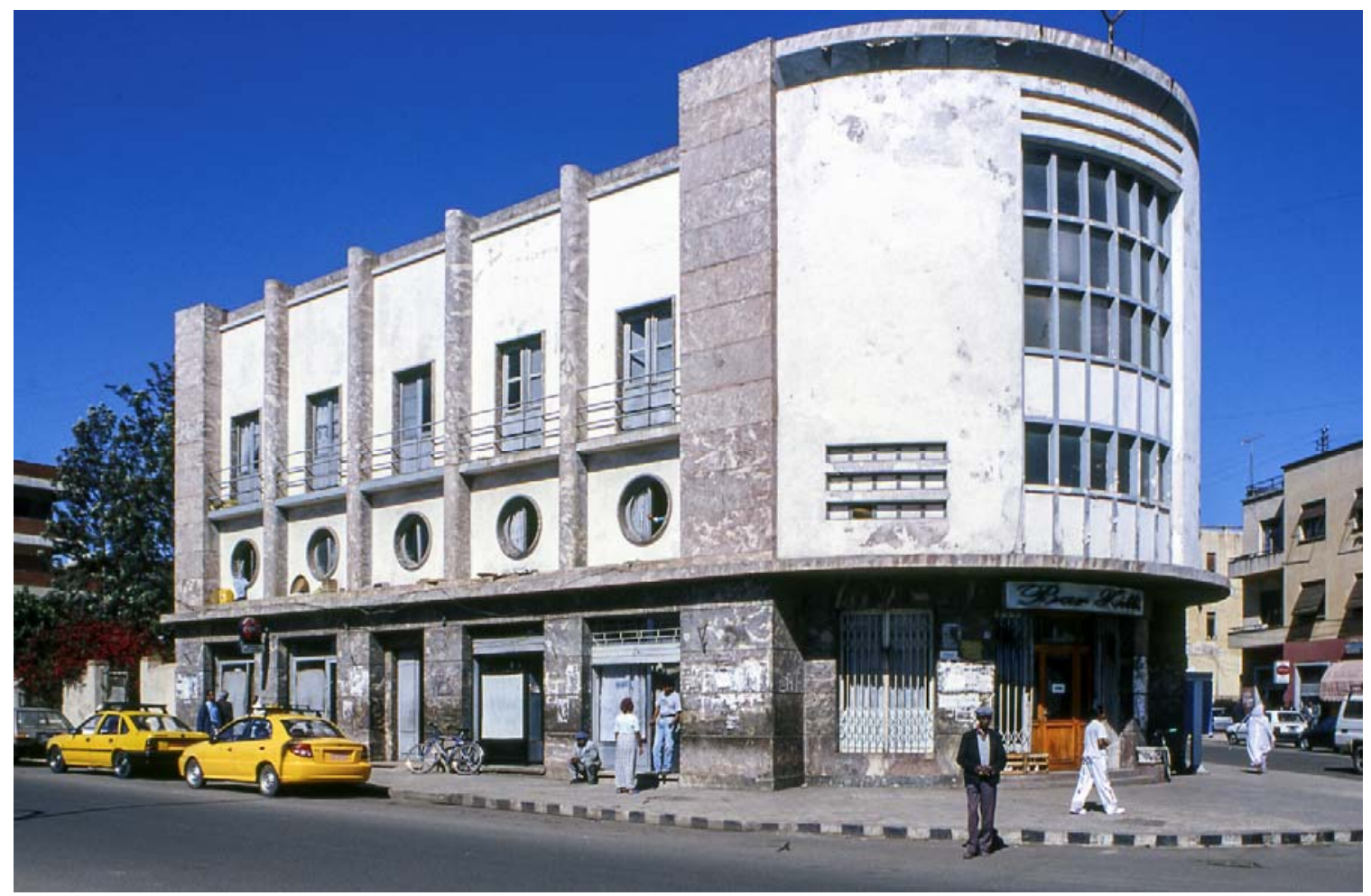

Fig. 3 ○ 2013, Maristella Casciato. Palazzo, costruito alla fine degli anni Trenta, noto come Zilli Bar dal nome dell'esercizio commerciale che occupa lo spazio d'angolo al piano terra. Il volume curvilineo accompagna con eleganza l'incrocio fra Semerat Avenue e Beirut Street.

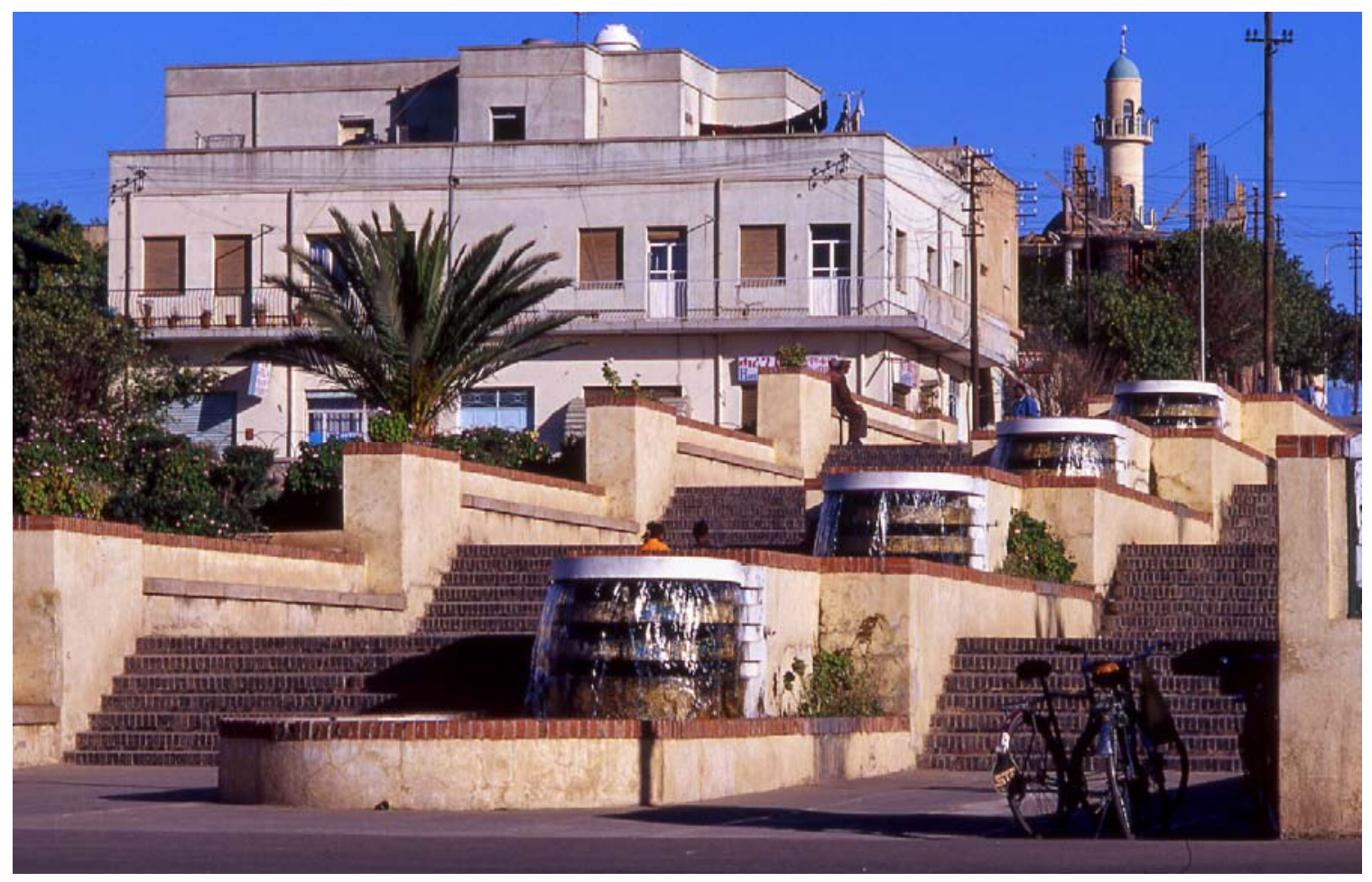

Fig. 4 ๑ 2013, Maristella Casciato. La fontana Mai Jah-Jah nel quartiere di Gheza Banda, 1938. 


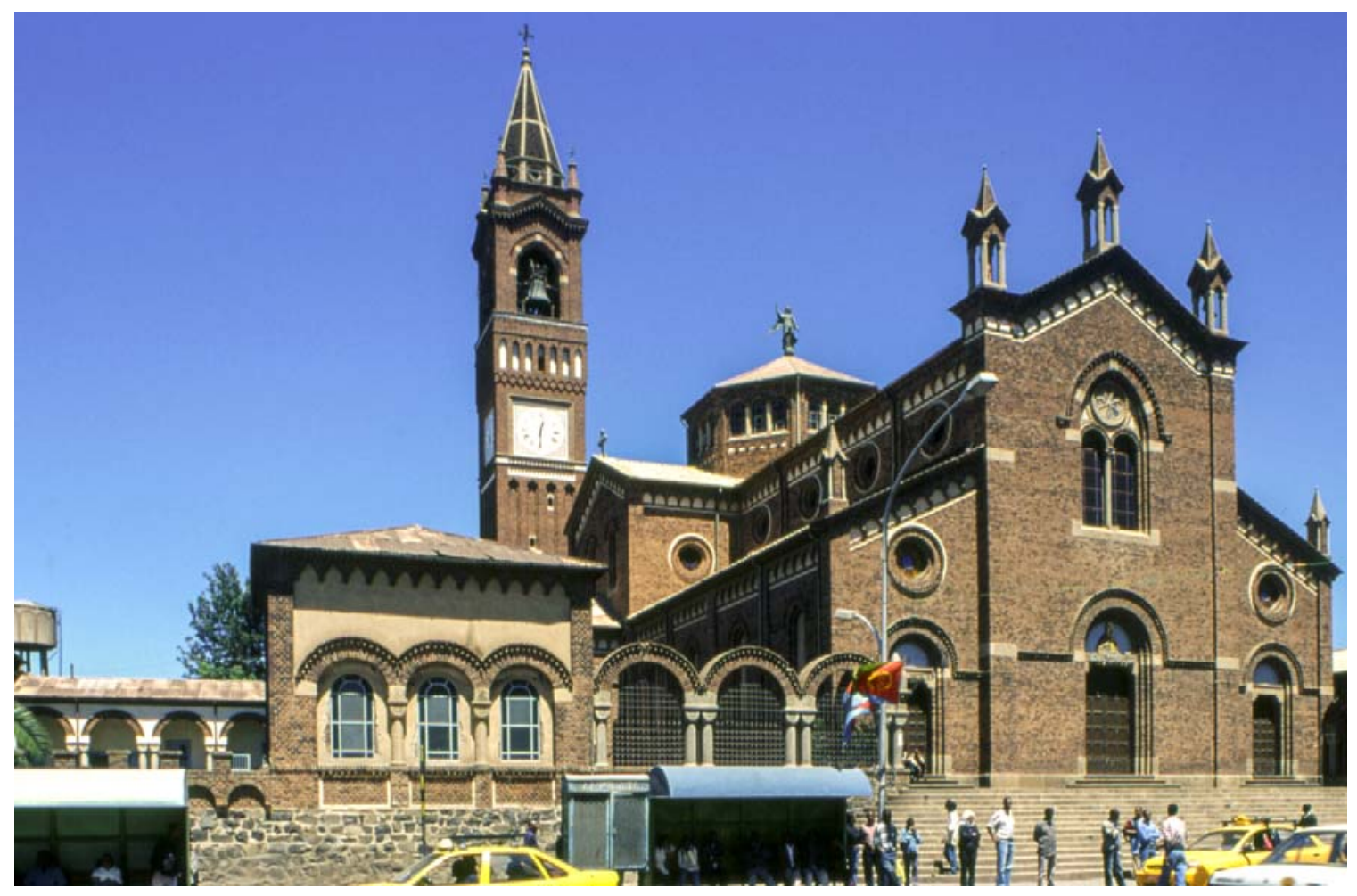

Fig. 5 ๑ 2013, Maristella Casciato. La cattedrale cattolica lungo viale Mussolini, oggi Harnet Avenue. La chiesa e l'adiacente missione cattolica, opera di Oreste Scanavini, risalgono alla prima metà degli anni Venti.

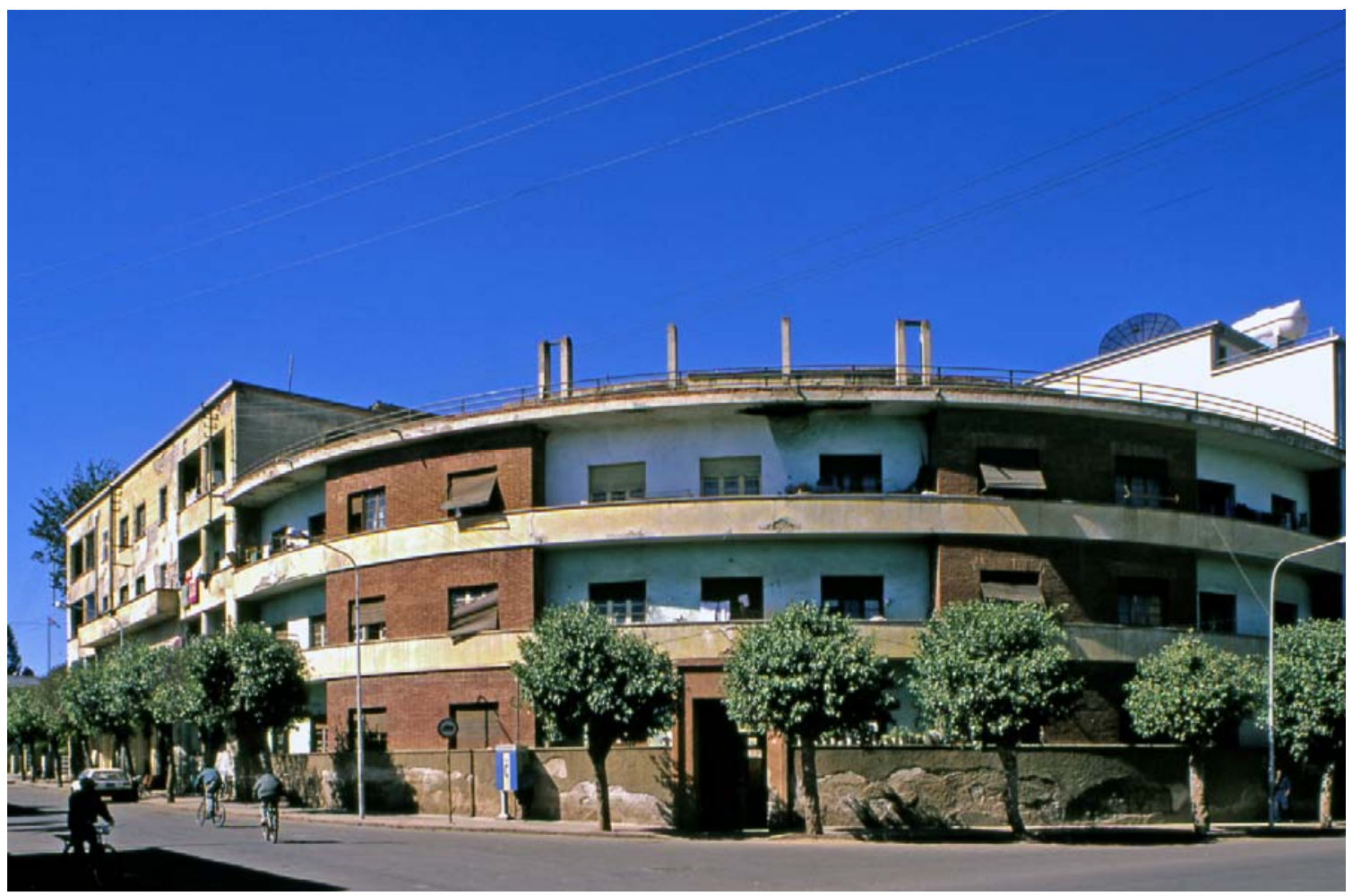

Fig. 6 ๑ 2013, Maristella Casciato. Edificio residenziale all'angolo fra Denden Street e Maryam Gmbi Street, progetto di Aldo D’Alessio e Carlo Marchi, 1938 ca. 


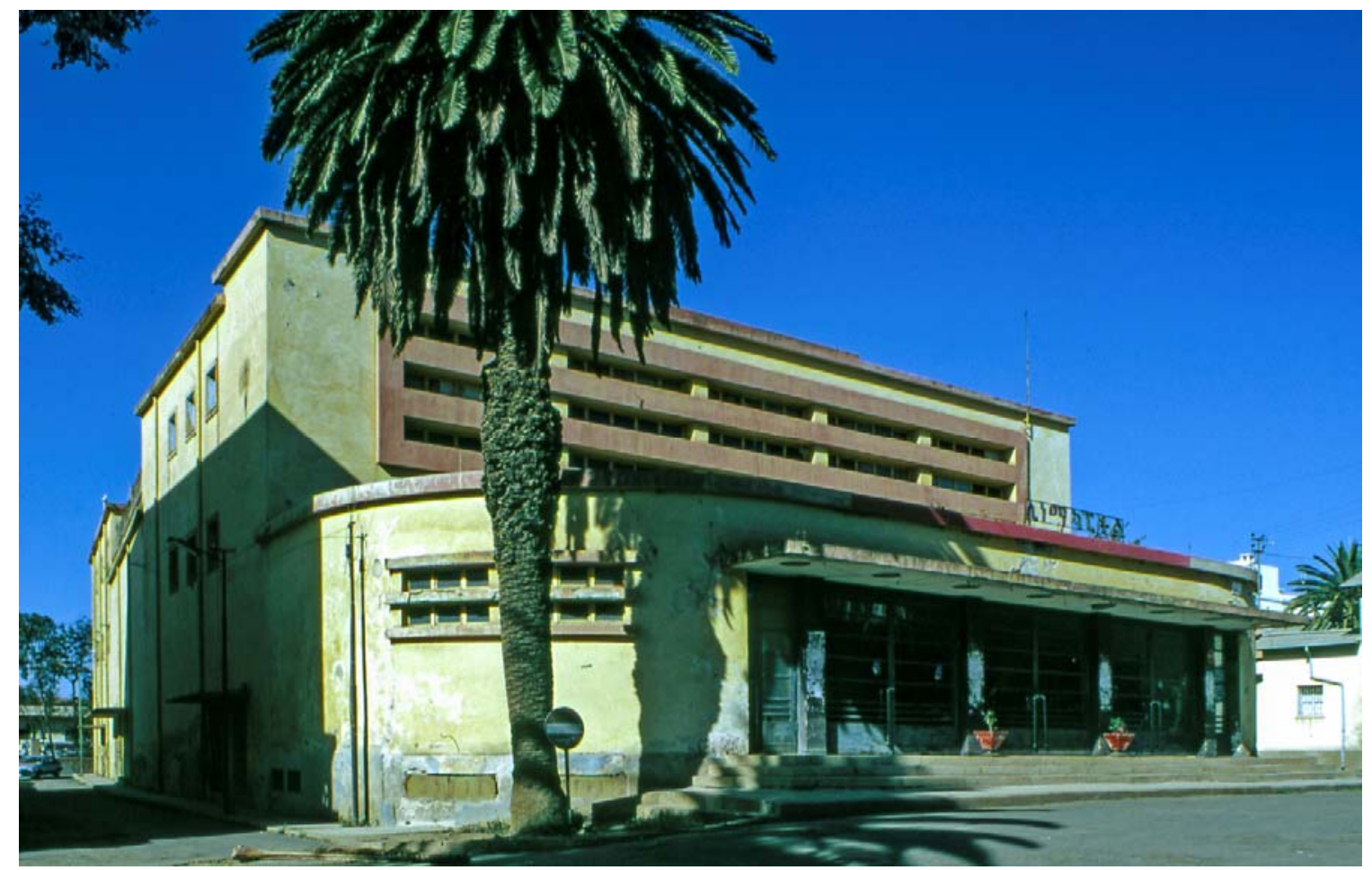

Fig. 7 ๑ 2013, Maristella Casciato. Cinema Augustus (oggi Capitol) su Denden Street. Progetto di Ruppert Saviele, 1938, parzialmente distrutto da un incendio, l'edificio fu riparato nel 1944.

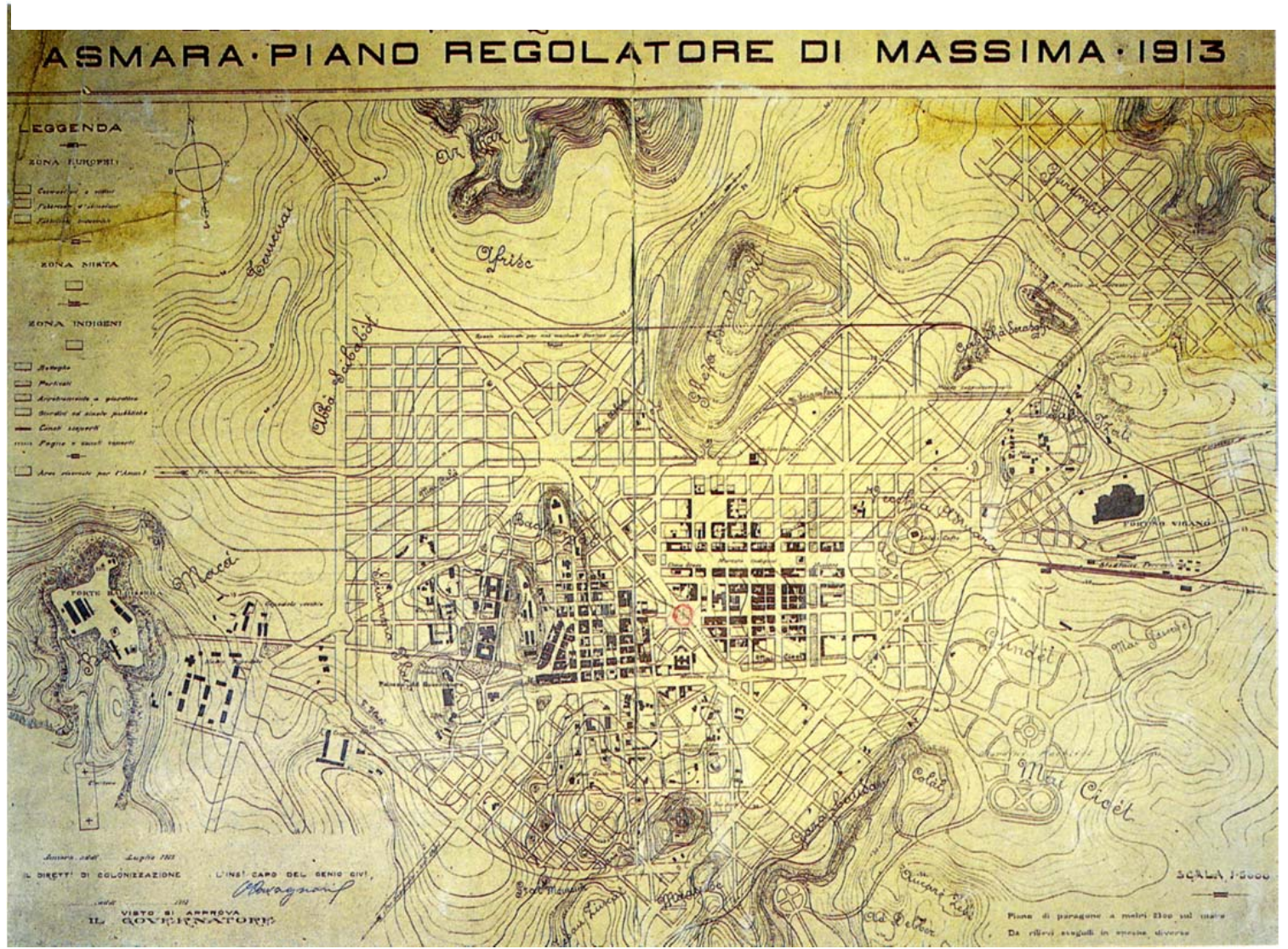

Fig. 8 @ 2013, Maristella Casciato. Odoardo Cavagnari, piano regolatore generale per Asmara, Ministero delle Colonie, 1913 (Archivio Centrale dello Stato, Roma) 


\section{Parole chiave}

colonie italiane in Africa, architettura razionalista, patrimonio moderno, Ferdinando Martini, Odoardo Cavagnari.

Maristella Casciato, architetto e storico dell'archiettura, è stata docente presso l'Università di Bologna. Ha ricevuto numerosi riconoscimenti internazionali: Fulbright Scholar, borsista all'Institut national d'historire d'art, Parigi, Mellon Senior Fellow presso il Canadian Centre for Architecture, Montréal.

Aree di ricerca: architettura olandese del XX. Secolo, teorie dell'architettura moderna e contemporanea, manualistica e letteratura tecnica, architettura italiana negli anni della ricostruzione postbellica.

Dal 2002 al 2010 è stata presidente di Do.Co.Mo. Mo. International, l'associazione per la documentazione, conservazione e tutela del patrimonio architettonico del XX. secolo. In quegli anni ha collaborato con il World Heritage Centre/Unesco, in particolare per il progetto d'iscrizione di Asmara nella Lista del Patrimonio Mondiale. Nel novembre 2012 è stata nominata Associate Director Research presso il Canadian Centre for Architecture, Montréal.

mcasciato@cca.qc.ca, maristella.casciato@unibo.it

\section{SUMMARY \\ From a Military Outpost to a Capital: Asmara, Italian Colony and Beyond}

The 'Primitive colony' of Eritrea, overlooking the shores of the Red Sea, or Mar Rosso, (from which comes 'Eritrea' or 'Red Land'), saw successive stages of Italian colonialism, with a robust increase during the years of Fascism. Asmara, a village that became the capital in 1891, still conserves an almost intact urban structure and architectural features from its half century of colonialism. The first expansion of Asmara was regulated by a scheme plan, approved in 1902, which included the area to the East of the original military outpost on the Mai Belà river. The grid pattern was marked by two major arteries, parallel to each other: the King's Way (il Corso del Re) and the Queen's Avenue (il Viale della Regina). After the space of a decade, Cavagnari's plan was the first to separate the European settlement from the indigenous zone. The advent of Fascism did not have an immediate impact in Eritrea; by then the capital had developed a well-integrated population. One is continuously surprised by testimonies of the residents in Asmara in the Thirties, which express a state of mind shared by both colonizers and colonized, describing the city as: 'beautiful [...] inhabited by a mixed race, Italians and Africans ... a lot of traffic, shops, cinemas and restaurants...' . It is commonplace to refer to 'good Italian people' (italiani brava gente), which is a most difficult viewpoint to abandon, and is also a most relevant perspective when considering the architectural patrimony of Asmara as a shared heritage. This paper aims to shed light on this mixture of narratives and to reread the modernity of Asmaran architecture as an added value to the contemporary history of the Eritrean nation. 